ISAHP 1996, Vancouver, Canada, July 12-15, 1996

\title{
AHP IN EVALUATION OF THE RELATIVE IMPORTANCE OF SUCCESS DIMENSIONS IN DEFENSE DEVELOPMENT PROJECTS
}

\author{
S. Lipovetsky \\ 1451 Overing St., \#19-D, Bronx, NY 10461, USA \\ 102170.2625@compuserve.com \\ A. Tishler, D. Dvir \\ Faculty of Management, Tel Aviv University, 69978, Israel \\ atishler@taunivm.tau.ac.il \\ A. Shenhar \\ U of MN, CDTL, 107 Lind Hall, 207 Church St. SE, Minneapolis, MN 55455, USA \\ shenhar@mailbox.cdtl.umn.edu
}

\begin{abstract}
A multidimensional approach was used to measuring the success of defense projects. Four dimensions of success were elaborated: meeting design goals, benefits to the customer, to the developing organization, and to the defense and national infrastructure. This study analyzed views of different stakeholders (customer, developing organization, and coordinating office within the Ministry of Defense) on the relative importance of these dimensions of success. Several approaches were applied: the classical AHP and its multiplicative form, Synthetic Hierarchy method of priority vectors evaluation, Analytic and Synthetic Hierarchy methods in Fuzzy interval estimation of the priorities. The results revealed that benefits to the customer is the most important success dimension, followed by meeting design goals. Surprisingly, other dimensions were relatively unimportant.
\end{abstract}

\section{Success Measures and Dimensions}

Data on 110 defense projects performed in Israel during the last twenty years was gathered using structured questionnaires and interviews. The questionnaires were filled out by at least three key personnel 'related to the project - one member of the customer (end-user) team, one member of the contractor team, and one representative of the Ministry of Defense coordinating office responsible for the budget and contractual issues. Thus, the different points of view of the major participants were reflected.

The questionnaires included data on twenty measures of success which were developed in earlier research (Dvir and Shenhar, 1992; Dvir et al., 1994; Tishler et al., 1996) and were adjusted to defense projects and the surveyed industries.

The measures were divided into four separate groups (dimensions). The first dimension was designated "meeting design goals" and refers to the contract that was signed with the customer. The second dimension refers to the benefits to the customer from the project's end-products. It includes measures for assessing the success in meeting technical and operational needs, as well as measures of customer satisfaction. The third dimension measures the benefits gained by the developing organization from executing the project, both in the short run and in the long run. The last dimension measures the benefits to the defense and national technological infrastructure gained from the project. The measures comprising each dimension are as follows:

- Meeting Design Goals: Functional specifications, Technical specifications, Schedule goals, Budget goals;

- Benefits to the Customer: Meeting acquisition goals, Meeting the operational need, Product entered service, Reached the end-user on time, Product had a substantial time for use, Product yields substantial improvement in user's effectiveness and/or capability, User is satisfied with:product;

- Benefits to the Developing Organization: Project yielded relatively high profit, Project opened new markets, Project created a new product line, Project developed a new technological capability, Project improved reputation; 
- Benefits to the Defense and National Infrastructure: Project developed a new technological capability, Project contributed to critical fields, Project maintains a flow of updated generations, Project decreases dependence on outside sources, Contribution to other projects.

In addition to providing a score for each success measure, all three assessors of a certain project were asked to rank the four dimensions of success according to their relative importance. This ranking procedure was done in two different ways. First, direct ranking, and second, ranking by pairwise comparison.

\section{Comparisons by Absolute Values}

Four success dimensions were ordered (in points, from 1 for the least important to 4 for the most important dimension) by "customer", "developer", and "Ministry of Defense" (MOD) kind of judges for 62, 71 and 44 projects, respectively. Even a simple analysis of the descriptive characteristics of the ranking data provides some insight into the issue of relative importance of success dimensions of defense projects.

Average results of the direct ranking procedure performed by the three different assessors (the end-user representative, the contractor representative and the coordinating office representative) clearly show that all three assessors agree that the most important success dimension is the second dimension - benefits to the customer. No such agreement exists regarding the other three dimensions. Nevertheless, the first dimension meeting design goals seems to be the second in importance, while only representatives of the coordinating office (who are in charge of building the defense technological infrastructure) think that the last dimensions is more important. Between the last two dimensions, most of the assessors think that the last dimension benefits to the national infrastructure is more important than the third dimension. Not surprisingly, the contractor representative rank (on the average) the third dimension - benefits to the contractor before the fourth dimension. However, by "customer" and by "MOD" opinion, the third dimension is the least important one: not only its mean value is the minimal among four dimensions, but even the range of particulars estimations does not exceed the point " 3 ") (when 4 is possible).

To estimate the success dimensions importance more precisely, using multiple comparisons, we applied the so called "Round Robin Tournament" procedure (Daniels, 1969; David, 1969; De Gray, 1968). The solution in this approach could be reduced to the first eigenvector of the matrix of not-centered second moments of points averaged by all projects.

Numerical results of this method are obtained for each group of experts. Comparison of these vectors with mean values shows that the weights of importance for four success measures are very consistent in estimation by simple averaging and by iterative Round-Robin procedure, and overail ranks of success dimensions are the same by both of the methods.

\section{Comparisons by Relative Values}

Another set of initial data was obtained by expert estimations of differences of the importance in pairwise of four success dimensions. For each pair the difference in importance was estimated in points from -3 to +3 for the least and most important first of items in comparison with the second of them. Then power transformation was used for presentation of absolute differences in the form of pairwise ratios (Lootsma, 1993; Triantaphyllou and Mann, 1994).

Separately for judges of "Customer", "Developer" and "MOD" kind, these ratios of pairwise comparisons were arranged into 3-way matrices of $4 x 4 x n$ order, where $4 \times 4$ plane corresponds to pairwise ratios Saaty matrix of each ith project in the set of all $n$ of them. The 3-way matrices of the pairwise ratio comparisons between four success dimensions contain 77 projects of "Customer", 91 projects of "Developer", and 54 projects of "MOD" estimations. The overall evaluation of success dimensions' importance by set of projects was performed by the following methods.

First, the so called logarithmic method, or multiplicative mode of the AHP (Saaty, 1994), was used for estimation priorities of four success measures by $4 \times 4 \times n$ matrices. This method could be described as the LSminimizing problem for the relative deviations (Lipovetsky and Tishler, 1994).

Numerical results obtained by ratio-scale estimations have more clearly expressed structure of preferences than results of difference-scale estimations. It means that big (small) weights by the Round-Robin evaluation become bigger (smailer) in multiplicative-mode evaluation. The ranks produced by these methods are the same for "Customer" and "MOD", but different for the two least important dimensions for "Developer" vectors of priority. 
The classical AHP method, applied to the data averaged by all $n$ projects was used too. For our three data sets the AHP produces priorities between those of the former two methods. Ranks of success for four dimensions are the same as in the previous methods for "Customer", but differs for two intermediate ranks (2 and 3) for "MOD" evaluation.

Another approach, Synthetic Hierarchy Method (SHM) (see Lipovetsky, 1996) was also applied. This approach corresponds to LS-minimization of deviations of priority parameters by all the elements of a $4 x 4 x n$ matrix of pairwise ratios. The results of SHM estimation are similar to the AHP results.

All the applied methods indicate that the most important dimension is Benefits to the Customer, and it is so by all "Customer", "Developer" and "MOD" evaluation.

To check the consistency of the results described above, we used the fuzzyfied data in pairwise comparison matrices. Their elements above the diagonal were fuzzyfied, and the elements below the diagonal (unestimated directly by experts) were constructed as in (Lipovetsky, 1994). Results demonstrate nonoverlapped ranges of weights obtained for left-right edges of all priority vectors for each kind of experts, and very clear picture of ranking for all success dimensions.

We can summarize that four success dimensions are ordered by their importance (preferability) by each of assessors as follows: Benefits to the Customer > Meeting Design Goals $>>$ Benefits to the Defense and National Infrastructure $>$ Benefits to the Developing Organization.

For all practical purposes, the success of defense projects, and possibly of all kinds of projects, should be evaluated only, or mostly, by the Benefits to the Customer and by Meeting Design Goals. That is, by measuring to what extent the customers are satisfied with the delivered product, and second, by measuring the level of success in meeting schedule, budget, technical and functional specifications.

\section{References}

Daniels, H.E. (1969), "Round-Robin Tournaments Scores", Biometrica, 56, 295-299.

David, H.A. (1969), The Method of Paired Comparisons, London: Griffin.

De Gray, R.J. (1968), "Notes on The Round Robin", Technometrics, 10, No. 4, 841-842.

Dvir, D. and A.L. Shenhar (1992), Measuring the Success of Technology-Based Strategic Business Units, Engineering Management Journal, 4, 4, 33-38.

Dvir, D., S. Lipovetsky, A. Shenhar and A. Tishler (1994), "Identifying Managerial Variables Critical to the Success of Projects: Multivariate Analysis of Large Data Sets", Faculty of Management, Tel Aviv University, Working Paper 6/94.

Lipovetsky, S. (1994), "The Aralytic and the Synthetic Hierarchy Processes: Fuzzy Applications", Faculty of Management, Tel Aviv University, Working Paper No. 7/94. .

Lipovetsky, S. (1996), "The Synthetic Hierarchy Method: An Optimizing Approach to Obtaining Priorities in the AHP", forthcoming in European Journal of Operational Research.

Lipovetsky, S. and A. Tishler (1994), "Linear Methods in Multimode Data Analysis for Decision Making", Computers and Operations Research, 21, No. 2, 169-183.

Lootsma, F.A. (1993), "Scale Sensitivity in the Multiplicative AHP and SMART", Journal of MultiCriteria Decision Analysis, 2, No. 2, 87-110.

Saaty, T.L. (1994), Fundamentals of Decision Making and Priority Theory with the Analytic Hierarchy Process. RWS Publications, Pittsburgh.

Tishler, A., D. Dvir, A. Shenhar and S. Lipovetsky (1996), "Identifying Critical Success Factors of Defense Development Projects: A Multivariate Analysis", forthcoming in Technological Forecasting and Social Change.

Triantaphyllou, E. and S. Mann (1994), "Some Critical Issues in Making Decisions with Pairwise Comparisons", Proceedings of the 3rd International Symposium on the Analytic Hierarchy Process, George Washington University, July 1994, Washington, DC. 